\title{
Classification of user postures with capacitive proximity sensors in AAL-environments
}

\author{
Tobias Grosse-Puppendahl, Alexander Marinc and Andreas Braun
}

TU Darmstadt, Karolinenplatz 5, 64289 Darmstadt, Germany

Fraunhofer IGD, Fraunhoferstr. 5, 64283 Darmstadt, Germany

t_groae@rbg.informatik.tu-darmstadt.de, \{alexander.marinc, andreas.braun\}@

igd.fraunhofer.de

\begin{abstract}
In Ambient Assisted Living (AAL), the context-dependent adaption of a system to a person's needs is of particular interest. In the living area, a fine-grained context may not only contain information about the occupancy of certain furniture, but also the posture of a user on the occupied furniture. This information is useful in the application area of home automation, where, for example, a lying user may effect a different system reaction than a sitting user. In this paper, we present an approach for determining contextual information from furniture, using capacitive proximity sensors. Moreover, we evaluate the performance of Naïve Bayes classifiers, decision trees and radial basis function networks, regarding the classification of user postures. Therefore, we use our generic classification framework to visualize, train and evaluate postures with up to two persons on a couch. Based on a data set collected from multiple users, we show that this approach is robust and suitable for real-time classification.
\end{abstract}

Keywords: AAL, capacitive proximity sensors, classification, user context

\section{Introduction}

Population aging in many industrial countries is posing various challenges to society, where a shrinking number of working persons has to care for an ever growing number of seniors. Assistive health-care applications, designed for elderly users, are trying to counteract the shrinking budgets in public health care. The paradigm of Ambient Assisted Living (AAL) represents methods, concepts, systems and services that unobtrusively support a person in daily life. User-centric technologies and concepts are integrated into the immediate environment of the person requiring assistance, and customized to individual needs and capabilities.

To support the adaptation of the environment to a user, it is necessary to determine an application context, particularly information about a person and any interaction with the environment. Typical examples include acquiring the location of a user within the premises or measuring relevant medical data, e.g. blood pressure. Determining a user's posture on furniture generates additional 
contextual information that can be used within AAL applications, for example safety features in home automation. If a user is lying on a bed, the system can deduct that he will remain there for a while, causing lighting and heating in adjacent rooms to be adjusted. If the user is suddenly sitting at night, the system may anticipate that the user is going to the toilet, thus activating dimmed lights to prevent tripping and falling.

The aim of this work is to classify the posture of a person using capacitive proximity sensors that are embedded into the living area. Classification refers to making a discrete observation, e.g 'a person is lying on a couch', derived from a set of incoming sensor readings. We have created a generic framework that is able to interface multiple sensor classes and classification methods. In this work we will present a system based on capacitive proximity sensors that can be unobtrusively integrated into existing furniture, while providing reliable information about the presence of a subject.

Capacitive proximity sensors use oscillating electric fields between an emitter and a ground electrode. The properties of an electric field change if a conductive object is brought into it. The human body, or bio mass in general, falls under this class, thus it is possible to unobtrusively detect a person or body parts of a person that approach such a sensing device [16]. Combining several sensors, we are able to fuse their outputs, in order to obtain information about the body posture.

We have chosen a couch in a living room to test our system. The generated context can be used for energy saving purposes, e.g. shutting off lighting in other rooms, but also for controlling ambient parameters like lighting and multimedia equipment that may react differently to sitting and lying persons. We have equipped an ordinary couch with eight capacitive proximity sensors and applied various classification techniques to the generated data. We have evaluated different classifiers by testing the prototype system on a diverse group of persons. Results show that such a system is reliably able to recognize various user postures, even if body mass and height differ strongly. Moreover, interviews performed with our test persons strongly indicate that the unobtrusive nature of capacitive proximity sensors will increase the user acceptance in actual applications, compared to camera-based systems. The interviews revealed that, using camera-based systems, people feel particularly observed and consider popular recent data leaks.

\section{Related Work}

Intelligent environments have been a research focus in the past decades [15]. Industry has started using developed technologies to link various technical devices in the living area. The property 'intelligent' refers to systems that are able to manipulate the environment without explicit user interaction, only using the implicit context a user generates, based on presence. To acquire this context, it is necessary to place various sensing devices in the living area. In order to manipulate the environment, actuators are required. Systems that include cognitive 
capabilities like perception, learning, reasoning, planning or executing tasks are denoted as Cognitive Technical Systems (CTS)[3].

Every element of a living area can be part of the CTS. For example, Beetz et al [4] have equipped a kitchen with several types of sensors, realizing various scenarios based on a robotic assistant for supporting activities of daily life. Indoor localization methods are an important part of many CTS. They allow the system to provide services like energy saving, home security and fall prevention. Methods include GSM triangulation [12] or capacitive sensors [13]. Capacitive sensors are applicable in various AAL-related scenarios, e.g. the detection of spine strain and subsequent user feedback on appropriate lying positions using an array of capacitive proximity sensors in a bed [7]. Kivikunnas et al [8] have equipped a couch with capacitive proximity sensors for future application in posture recognition.

The fusion and interpretation of data generated by various sensors is one of the main application areas of machine learning [10]. Specifically they can be used to map the continuous input sets to discrete classes. Amoretti et al describe example applications in AAL [1]. As proof-of-concept for our posture recognition in AAL environments we have tested the system by building a prototype based on a couch augmented with capacitive proximity sensors.

\section{User posture classification}

\subsection{Sensors}

By using capacitive proximity sensors, we can measure the proximity of a person's body. A typical capacitive sensor consists of an electrode, emitting an electric field that oscillates at $10-200 \mathrm{KHz}$ and a receiving electrode that measures certain properties of this electric field. Other designs, like ours, are based on a single electrode that creates a field with other electric potentials within reach, for example a ground node. When body mass enters this electric field, the displacement current, measured in the emitting node, changes. This effect makes it possible to detect the proximity of body mass[16]. Furthermore, it is possible to gather information about the distance of the object entering. If an object is uniformly sized, the displacement current will increase when it is brought closer to an electrode. The operating range and resolution of this sensor type strongly depends on the electrode size, material used, emitter frequency and emitter voltages, resulting in achievable distances between a few nanometers and several meters [2]. The generated electric fields will progress through any non-conductive material. Thus it is possible to install these sensors unobtrusively, e.g. underneath the upholstery of wooden elements or furnishing. This makes them less prone to mechanical influences in the operating and setup time, as well as hiding active system parts from the user allowing a free external design of the furnishing. 


\subsection{Preprocessing \& Feature extraction}

The sensors deliver continuous signals, which are sampled with a low frequency, e.g. $10 \mathrm{~Hz}$, and normalized to an interval between 0 and 1 . In the next step, overlapping short-time windows (e.g. with a length of 1 second), containing samples from all sensors, are built. Due to the high amount of data contained in a window, a direct classification is computationally complex or even impossible. Thus, we must focus on relevant information for classification, the features of a short-time window.

Typical features for user posture classification are the empirical mean and the standard deviation of a short-time window. For example, we may extract the empirical mean from each sensor and use it for classification. A feature vector $X_{i}=\left(x_{1}, \ldots, x_{n}\right)^{T} ; X_{i} \in \Re^{N}$ consists of all extracted features, whereas the feature space contains all these vectors. In order to be able to uniquely identify the classes in the classification step, the extracted features must build a compact and bounded subspace for each class [11].

\subsection{Classification}

We aim to recognize a discrete class $z_{k}, k \in\{0,1, \ldots, K\}$ from the extracted feature vector $X_{i}$. A class may be represented by a statement like'one person is sitting at the right side of the couch' that reflects an observation of the actual system.

To cope with the complex task of classification, we need to learn from experience, making use of an annotated training set of feature vectors and the corresponding classes. The main goal of classification is to reliably identify a class for unknown feature vectors. Thus, generalization is a very important property of a classifier. In the following, we will present three suitable classifier models for user posture classification.

Naïve Bayes models are very popular models in machine learning applications due to their simplicity and computational efficiency. The classifier assigns the most likely class $z_{k}$ to a given feature vector $X_{i}$. The classifier's model structure is simplified by the assumption that the distributions of all features for a specific class are conditionally independent. This assumption of conditional independence of features is often seen as a weakness of the Naïve Bayes model. However, this property makes the Naïve Bayes model very fast and efficient for testing and evaluation purposes [5].

Unlike the Naïve Bayes model, Decision tree classifiers can overcome the problem of conditional dependence of features. A decision tree consists of nodes, which represent logic rules and leafs, which represent the final decision, as shown in figure 1 at the right. Classifications are made by traversing the tree structure, evaluating a decision function in each node, until a leaf is reached. In practice, binary decision trees with threshold decision functions for a single feature are commonly used (e.g. $x_{j}<=r ; x_{j}, r \in \Re$ ). The advantage of decision trees is the input-dependent traversal of its nodes. Decision trees can be built with 

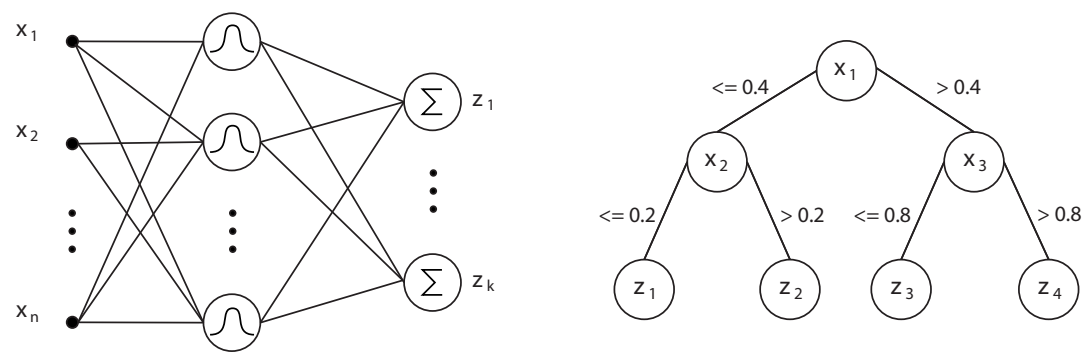

Fig. 1. Left: Layout of an RBF network. Right: An example decision tree

algorithms like the C4.5 algorithm, which selects the feature with the highest information gain for each decision node $[9,5]$.

Radial basis function (RBF) networks, as shown in figure 1 at the left, are a specialization of artificial neural networks and are often applied on classification problems. RBF networks consists of three layers, one input layer, a hidden layer with radial activation functions and an output layer, that implements a weighted sum over the hidden unit's outputs. The number of chosen basis functions is essential for the network's generalization abilities. RBF networks, or artificial neural networks in general, face the problem that their output behaviour is often not easy to comprehend, especially when a transparent functioning is required $[9,6]$.

\section{The smart couch scenario}

\subsection{SenseKit: A generic approach}

Since there are numerous scenarios for applying user posture classification, we have developed a generic framework called SenseKit for posture classification tasks. Different scenarios, e.g. posture-detecting chairs or beds require different types and numbers of capacitive sensors, in order to reliable detect the required postures. Sensekit tackles these classification tasks and provides additional functionality, most notably visualization and evaluation of the processing pipeline. Sensekit is based on a configurable dependency injection framework that allows all components (classifiers, sensors, feature extractors, etc.) to be dynamically combined, corresponding to the individual application scenario.

Apart from SenseKit's classification and digital signal processing abilities it also implements training and visualization components. Sensor readings, as well as the final classification results, may be visualized and presented in an effective way. We have integrated various machine learning algorithms into our framework. Most algorithms are adapted from the WEKA Machine Learning Project [14]. Moreover, we integrated WEKA's explorer into our framework, a comprehensive tool that provides functionality to evaluate recorded training data. 

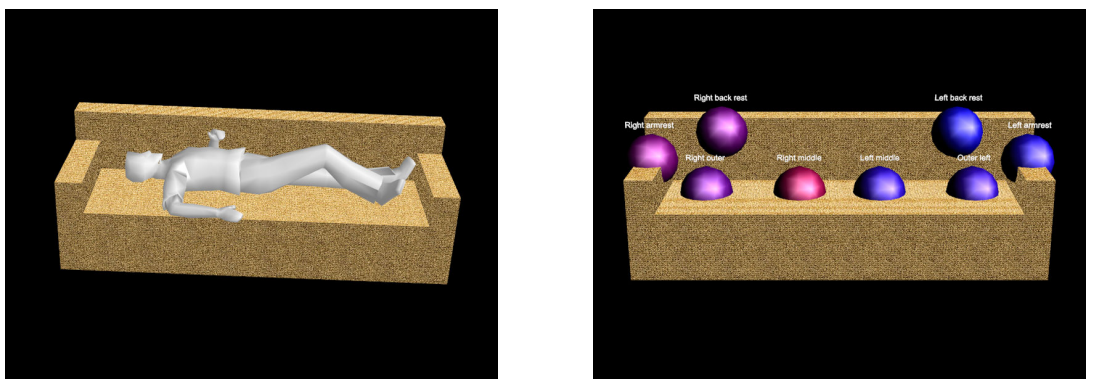

Fig. 2. Left: Visualization of a classification. Right: Visualization of sensor readings

Our prototype is an ordinary couch augmented with capacitive proximity sensors hidden underneath the upholstery. In order to prove our methodology, we intended to classify a high amount of potential postures, various sitting and lying positions (2 at the left) for one and two persons. We deemed eight sensors to be sufficient in establishing a good data basis for classification of the various postures. The final design consists of two sensors, placed underneath both armrests, two sensors in the back rests and another four sensors underneath the sitting area, as shown in figure 2 at the right. Even though the sensors are up to 15 centimeters away from the user and covered by upholstery and wood (see 3 ), we are still able to retrieve good measurements of body mass proximity, supported by the fact that some electrodes have pressure applied to them, causing a geometric deformation that also affects the output signal.
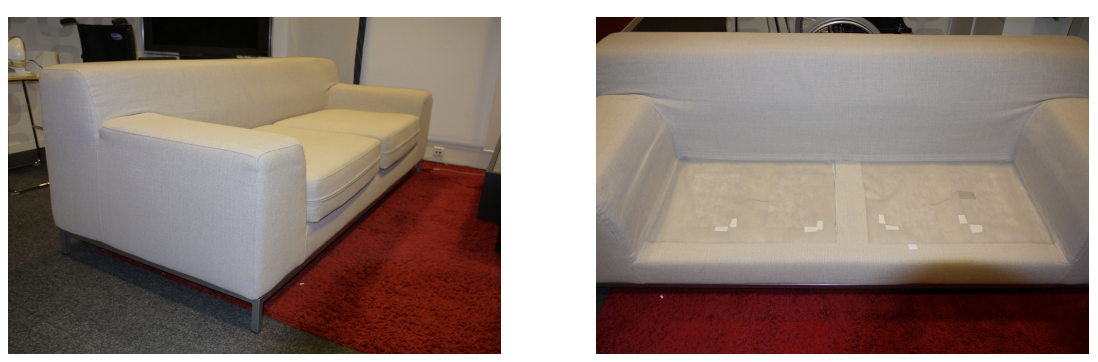

Fig. 3. An ordinary couch has been equipped with capacitive proximity sensors that have been set up under the upholstery and wooden elements

In order to determine a suitable test set, we are distinguishing nine different possible postures on a couch that can be performed by one or two persons. The 18 test persons were given simple written instructions to perform the desired postures. The persons performed all postures, relaxed and without restrictions in their movements, for approx. 30 seconds. Similar postures were always interrupted by unrelated postures. The data set contains a training set, with data 
from 9 test persons, and a test set with data of another 9 persons. Both, the training and test data set, were recorded on different days with different test persons. Additionally, we recorded body weights and sizes (figure 4), since those are the main properties affecting sensor measurements. Our training set consists of 2829 instances (about 24 minutes), whereas our test set consists of 2312 instances (about 20 minutes).
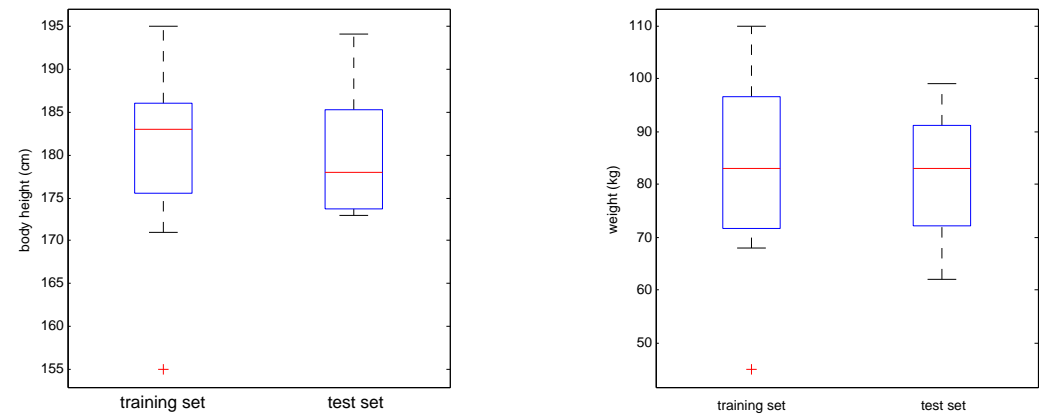

Fig. 4. A box plot of body heights and weights in our data set. The blue box denotes data from lower to upper quartile, the red dash denotes the median and red crosses mark outliers.

In the regarded scenario, we use the empirical means of our eight sensors, which are extracted from a short-time window, as feature vector. Overlapping short-time windows are passed to the classifier every second, containing the last 2 seconds of sensor readings.

\subsection{Evaluation}

Three classifiers were evaluated on our data set. We evaluate the performance of the Naïve Bayes classifier, decision trees and RBF networks. To measure the performance, we consider the metrics of precision and recall. As each sensor has individual characteristics, e.g. caused by different electrode sizes, the evaluation results are not symmetrical concerning the geometry of the couch. Furthermore, each sensor produces an individual amount of noise that has to be taken into account. The performances of the three classifiers are shown in table 1 .

Our evaluation results show that the Naïve Bayes model does deliver inferior results compared to more sophisticated models, such as RBF networks. This fact is mainly caused by the very strong assumption of conditional independence, which is not satisfied in user posture classification scenarios. However, the Naïve Bayes model provides sufficiently precise results, as well as efficient training and data analysis. We retrieve an overall recall of $92.2 \%$ and a precision of $90.6 \%$. 
Naïve Bayes $\mid$ Decision Trees $\mid$ RBF network

\begin{tabular}{l|l|l|l|l|l|l}
\hline Class & Prec & Rec & Prec & Rec & Prec & Rec \\
\hline sitting outer left one person : OL & 0.92 & 0.97 & 1.0 & 0.84 & 1.0 & 0.99 \\
\hline sitting middle left one person : ML & 0.99 & 0.60 & 0.99 & 0.90 & 0.98 & 0.88 \\
\hline sitting outer right one person : OR & 1.0 & 0.78 & 1.0 & 0.63 & 1.0 & 0.96 \\
\hline sitting middle right one person : MR & 0.96 & 0.93 & 0.93 & 1.0 & 1.0 & 0.95 \\
\hline lying head right one person : LR & 0.77 & 1.0 & 1.0 & 0.89 & 0.92 & 1.0 \\
\hline lying head left one person : LL & 0.85 & 1.0 & 0.7 & 0.95 & 0.98 & 0.99 \\
\hline two persons sitting together : TT & 0.77 & 1.0 & 0.95 & 1.0 & 0.87 & 1.0 \\
\hline two persons sitting gap : TG & 1.0 & 0.99 & 0.98 & 0.64 & 1.0 & 0.99 \\
\hline no person : NP & 1.0 & 0.92 & 0.66 & 1.00 & 1.0 & 1.0 \\
\hline \hline Weighted average & 0.92 & 0.91 & 0.91 & 0.87 & 0.98 & 0.97
\end{tabular}

Table 1. Evaluation results for the three classifiers

The evaluation of decision trees, built with the C4.5 algorithm, shows similar results as the Naïve Bayes model. Classes like 'two persons sitting together with a gap' were sometimes classified as lying postures. Moreover, many activities with lower sensor measurements, e.g. caused by a low body weight, have been classified as 'no person', resulting in a poor precision of $66.4 \%$ for this particular class. The overall recall is $87.3 \%$, whereas the overall precision is $90.7 \%$.

Table 2 shows the confusion matrix of an RBF network, evaluated on our test set. We can see that some sitting postures on the right of the couch have been classified as lying head right postures, leading to a lower precision for this class. Moreover, we retrieve a low recall for sitting middle left postures, as they are often misclassified as sitting together postures. However, in general, RBF networks perform very well on the test set with an overall recall of $97.5 \%$ and a precision of $97.2 \%$. Furthermore, the determined clusters and their corresponding weights indicate that all sensors contribute equally to classification.

\begin{tabular}{l|l|l|l|l|l|l|l|l|l|l|l} 
& $\mathrm{OL}$ & $\mathrm{ML}$ & $\mathrm{OR}$ & $\mathrm{MR}$ & $\mathrm{LR}$ & $\mathrm{LL}$ & $\mathrm{TT}$ & $\mathrm{TG}$ & $\mathrm{NP}$ & Prec & Rec \\
\hline sitting outer left one person : OL & 296 & 1 & 0 & 0 & 0 & 2 & 1 & 0 & 0 & 0.99 & 0.987 \\
\hline sitting middle left one person : ML & 3 & 227 & 0 & 0 & 0 & 0 & 28 & 0 & 0 & 0.983 & 0.88 \\
\hline sitting outer right one person : OR & 0 & 0 & 253 & 0 & 11 & 0 & 0 & 0 & 0 & 1.0 & 0.958 \\
\hline sitting middle right one person : MR & 0 & 0 & 0 & 243 & 12 & 0 & 0 & 0 & 0 & 1.0 & 0.953 \\
\hline lying head right one person : LR & 0 & 0 & 0 & 0 & 260 & 0 & 0 & 0 & 0 & 0.919 & 1.0 \\
\hline lying head left one person : LL & 0 & 3 & 0 & 0 & 0 & 254 & 0 & 0 & 0 & 0.981 & 0.988 \\
\hline two persons sitting together : TT & 0 & 0 & 0 & 0 & 0 & 0 & 197 & 0 & 0 & 0.872 & 1.0 \\
\hline two persons sitting gap : TG & 0 & 0 & 0 & 0 & 0 & 3 & 0 & 212 & 0 & 1.0 & 0.986 \\
\hline no person : NP & 1 & 0 & 0 & 0 & 0 & 0 & 0 & 0 & 306 & 1.0 & 1.0
\end{tabular}

Table 2. Confusion matrix for the RBF network classifier 
We can conclude that RBF networks are a robust classifier model with a high accuracy for user posture classification in our scenario. The generalization abilities of this classifier are coping well with the variation of body heights and weight of the different test persons. We have identified a decent generalization ability as an essential requirement for classifiers in user posture classification.

\section{Summary}

\subsection{Conclusions}

We have shown that capacitive proximity sensors are well-suited to give robust and reliable information about a user's context, proven in an evaluation with 18 different test persons of diverse body height and weight. Using only eight sensors in our couch example we have achieved a reliability of more than $97 \%$ in eight different postures using RBF network based classifiers. The classification based on machine-learning methods is easily implemented, trained and can be visualized by using the created SenseKit framework. We have achieved a finegrained and reliable detection of user application context that can be used by intelligent systems to control the environment.

\subsection{Future Work}

Open issues are the reliable detection of nearly similar postures, e.g. one person lying and two persons sitting. Most issues related to this topic can be solved by simply using more sensors. Even though SenseKit is supporting this the higher costs and complexity of the used hardware are undesirable. We intend to test other physical sensor configurations that could achieve better results as our current prototype. However, a well-defined theory and methodology, that describes the ideal distribution of sensors within the furniture, based on number of sensors and desired posture classes, is highly desired. Given the nature of capacitive proximity sensors and the highly complex distribution of electric fields, another option would be to use a simplified model to simulate the sensor values within the furniture and apply optimization strategies to achieve a good sensor configuration. We are planning to integrate some of this functionality in future versions of our easily extendable SenseKit. Another topic of interest is testing our system on different types of furniture and integrating other types of sensors that might provide more diverse applications while achieving the same reliable results.

\section{Acknowledgments}

We would like to thank the students of TU Darmstadt and the employees of Fraunhofer IGD that lent us their sitting and lying abilities for the evaluation. 


\section{References}

1. Amoretti, M., Wientapper, F., Furfari, F., Lenzi, S., Chessa, S.: Sensor data fusion for activity monitoring in ambient assisted living environments. In: Sensor Systems and Software, pp. 206-221. International Conference on Wireless Sensor Network (WSN) Systems and Software 2009 (2009)

2. Baxter, L.: Capacitive Sensors: Design and Applications. IEEE Press (1996)

3. Beetz, M., Buss, M., Wollherr, D.: Cognitive technical systems what is the role of artificial intelligence. In: KI 2007: Advances in Artificial Intelligence, pp. 19-42. Springer Berlin, Heidelberg (2007)

4. Beetz, M., Jan, B., Kirsch, R., Maldonado, A., Mller, A., Rusu, R.B.: The assistive kitchen a demonstration scenario for cognitive technical systems. In: in Proceedings of the 4th COE Workshop on Human Adaptive Mechatronics (2007)

5. Bishop, C.M.: Pattern Recognition and Machine Learning (Information Science and Statistics). Springer-Verlag New York, Inc. (2006)

6. Buhmann, M.D.: Radial Basis Functions: Theory and Implementations. Cambridge University Press (2003)

7. Hamisu, P., Braun, A.: Analyse des schlafverhaltens durch kapazitive sensorarrays zur ermittlung der wirbelsulenbelastung. In: Proceedings of the Conference of Ambient Assisted Living 2010. VDE Verlag GmbH (2010)

8. Kivikunnas, S., Strmmer, E., Korkalainen, M., Heikkil, T., Haverinen, M.: Intelligent furniture and their ubiquitous use scenarios. In: Proceedings of the AALIANCE conference (2010)

9. Kotsiantis, S.B.: Supervised machine learning: A review of classification techniques. In: Proceeding of the 2007 conference on Emerging Artificial Intelligence Applications in Computer Engineering: Real Word AI Systems with Applications in eHealth, HCI, Information Retrieval and Pervasive Technologies. pp. 3-24. IOS Press (2007)

10. Mitchell, T.M.: Machine learning and data mining. Commun. ACM pp. 30-36 (1999)

11. Niemann, H.: Klassifikation von Mustern. Universität Erlangen-Nürnberg, Lehrstuhl für Mustererkennung (2003)

12. Otsason, V., Varshavsky, A., LaMarca, A., Lara, E.d.: Accurate gsm indoor localization. In: IN THE PROC. OF UBICOMP 2005. pp. 141-158 (2005)

13. Steinhage, A., Lauterbach, C.: Sensfloor(r): Ein aal sensorsystem fr sicherheit, homecare und komfort. In: Proceedings of the Ambient Assisted Living Congress 2008 (2008)

14. University of Waikato: Weka machine learning project (May 2011), http://www . cs.waikato.ac.nz/ml/weka

15. Wren, C.R., Sparacino, F., Azarbayejani, A.J., Darrell, T.J., Starner, T.E., Kotani, A., Chao, C.M., Hlavac, M., Pentland, A.P.: Perceptive spaces for performance and entertainment: Untethered interaction using computer vision and audition. Applied Artificial Intelligence pp. 267-284 (1997)

16. Zimmerman, T.G., Smith, J.R., Paradiso, J.A., Allport, D., Gershenfeld, N.: Applying electric field sensing to human-computer interfaces. In: Proceedings of the SIGCHI conference on Human factors in computing systems. pp. 280-287. CHI '95 (1995) 